\title{
THE PRINCIPLE OF EQUIVALENCE OF ENERGY SPENT ON PROCESS AND OF STEP OF TIME
}

\author{
Emil V. Veitsman $1 \bowtie$ (iD) \\ ${ }^{1}$ Veitsman’s Science project, 28 deparment, 5 Klimashkin Str., 123557, Moscow.
}

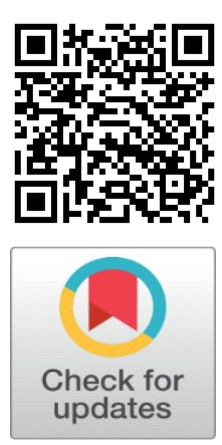

\section{ABSTRACT}

It was formulated the physical principle of the equivalence of the energy expense during the process of the Universe spreading and of the step of time. It was also shown that physical time is a material quantity connected with expending our Universe at constant velocity. A parallel was carried out between the increase of the size of the drops and bubbles and the expending of the Universe. The above principle is in full agreement with SR and GTR. It was shown as well that physical time could quantize.

Keywords: Space, Time, Universe, Principle of Equivalence, Power, Relativity

Received 15 September 2021

Accepted 15 October 2021

Published 31 October 2021

\section{CorrespondingAuthor}

Emil V. Veitsman, evveitsman@gmail.com

DOI

10.29121/granthaalayah.v9.i10.2021 .4320

Funding: This research received no specific grant from any funding agency in the public, commercial, or not-for-profit sectors.

Copyright: (C) 2021 The Author(s). This is an open access article distributed under the terms of the Creative Commons Attribution License, which permits unrestricted use, distribution, and reproduction in any medium, provided the original author and source are credited.

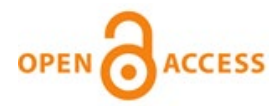

\section{INTRODUCTION}

As we know from special relativity (SR), there is the principle of equivalence of the energy $\mathrm{E}$ and the mass "m" of the body, i.e., $\mathbf{m c}^{2}$.We know as well the nature of the energy and body mass however, we know nothing about the nature of physical time. The physical parameter $\mathrm{T}$ is the most important physical one in science but peoples did not know up to $21^{\text {th }}$ century about its origin.

In Veitsman (2019) it was exposed the connection between the long-ranged interaction forces of the dimension $\mathbf{n}=1,2, \ldots, \mathbf{i}$ and the dimension of the isotropic space $\mathbf{m}=1,2, \ldots, \mathbf{j}$. It was shown: $\mathbf{j}=\mathbf{n}+1$ (Veitsman's theorem on spaces and forces forming these spaces). In Veitsman (2019) was studied as well weakly anisotropic spaces. It was established that such object being as an ellipsoid could not be in equilibrium. Here we must add the following. It was shown in Veitsman (2002) that drops of liquid and bubbles of gas cannot be in equilibrium. The smaller the sizes of the drops and bubbles the further they from equilibrium. In such case, the pressure in the drop or bubble $p$ (spherical form) equals Nitza et al. (1977) :

$P=2 \sigma / R$,

where $\sigma$ is surface tension; $R$ the radius of drop or bubble,

There are electromagnetic attractive interactions between microparticles in bubbles and drops. Our Universe can be by sphere form but the main interaction in it between masses is gravitationally, i.e., also attractive interaction. Bubbles and drops increase like the Universe. Here we have a certain analogy between them - gravitation is the result of the surface curvature of given volume.

In Veitsman (2021) we shown that the physical time was generated by the energy expense during the process of the expansion of our Universe. This 
expansion must be stationary. The energy expense at the expansion of our Universe is much more the sum expenses of all processes in our space-time. If the expansion of the Universe is not stationary, there will be information chaos in it and, as a result, the breach of the causal connection in the system under study. Then we can formulate the principle of the equivalence between of energy expense during the physical process and the step of time, primarily the energy expense and the step of time in our Universe. It is the main problem, which we have to solve in this scientific work.

\section{MATERIALS AND METHODS}

Now we can write down using stated above and in Veitsman (2019), Veitsman (2002), Nitza et al. (1977), Veitsman (2021):

$$
\Delta E=M \Delta T
$$

where $\mathrm{M}$ is a Lorentz Invariant, a certain analog of power.

However, if the parameter M in F.2 is the Lorentz invariant, then the parameters $\Delta E$ (the expense of energy on a certain process) and the parameter $\Delta T$ (the step of time) are equivalent. As a conclusion, formula (2) expresses an equivalence between energy and time in nature.

The above formula (in essence a hypothesis) does not contradict neither SR, nor GR. Let's start with SR.

Let us write down the expression for the transformation of the time interval $\Delta t_{0}$ in the frames of Lorentz transformations having remembered that they are correct only in inertial reference systems, i.e., moving uniform and rectilinearly.

$$
\Delta t=\frac{\Delta t_{0}}{\sqrt{1-\left(\frac{v^{2}}{c^{2}}\right)}}
$$

where $\Delta t_{0}$ is the interval of time for an observer being in the laboratory frame of reference, let it be fixed "(it is also the proper time in the moving reference frame)"; $v$ the speed of the moving frame of reference; $c$ the speed of light in vacuum.

Let $\Delta t_{0}$ be a unit time step when $v_{0}=0$, then $\Delta t$ is the step of time when the speed of the reference frame is equal to $v$.

I emphasize once more that the velocity of the moving system is uniform and rectilinearly

Now let us turn to the transformation formula of spending the energy $\Delta E$ of the system in dependence from the velocity $v$ :

$$
\Delta E=\frac{\Delta E_{0}}{\sqrt{1-\left(\frac{v^{2}}{c^{2}}\right)}}
$$

Taking into account ( $2-4)$ and considering power as a relativistic invariant, we came to the equivalence of energy and time (let us remember $\mathbf{m c}^{2}$ ).

Let us now come to General Relativity, in particular, to cosmology. Friedman's solutions of Einstein's equation lead to the case of homogeneous and isotropic Universe for different cases of its changes during time beginning from its static state 
and finishing by stationarity of different kinds. If the object under study is in static existence, then cosmology constant $\Lambda=\Lambda_{c}$ reaches of its critic value. In turn, certain coefficient $k$ from the solutions of Einstein's equation has to be equal unit. It means that this equation is found for a sphere. There is no the parameter "time" in it, and, i.e., there is no any time in the system studied. Here we have in view the general time for the all Universe.

In case, when $\Lambda>0$ and $k \leq 0$, the Universe monotonously expends itself, if its radius does not reach great values. At last, if $\Lambda$ is more than $\Lambda_{c}$ slightly, i.e., more than certain critic value, then the velocity of the Universe expending does not change during certain time. That is, the process of the evolution of our Universe may be stationary on that or another line segment of its existence but at this step of the universal time will be constant. However, this step has possibility to change itself in those or others subspaces e.g., according to the formula (3) and not only according to it.

Here we must draw a parallel between hydro- aerodynamics, and physical time. If the process of the gas or liquid moving is near to equilibrium then a following dependence takes a place for the moving of the viscous liquid and gas for a stationery case according to thermodynamics of irreversible processes Veitsman (2021):

$$
P_{i j}=P_{j i}=-\eta\left(\frac{\partial v_{i}}{\partial z_{j}}+\frac{\partial v_{j}}{\partial z_{i}}\right)+\left(\frac{2}{3 \eta}-\xi\right) \delta_{i j} \operatorname{divv},(\mathrm{i}, \mathrm{j}=1,2,3)
$$

Where $P_{i j}=P_{j i}$ is the stress tensor of the viscosity forces; v velocity vector;

$v_{i}, v_{j}$ the components of $\mathrm{v} ; \eta$ the coefficient of viscosity; $\xi$ the coefficient of bulk viscosity; $\delta_{i j}$ Kronecker symbol,

Expression (5) is correct only for the laminar flow of liquid and gas. If our systems under study are far from equilibrium, then we have turbulent flows, i.e., there is chaos takes a place, e.g., tornado.

In turn, if Universe is expending uniformly and enough slowly, then there are causal relationships in it. However, if the Universe is expending unevenly and very quickly, then these relationships will be broken, and information chaos will take a place in our system under study.

At last, the once more important conclusion follows from the above formula (2): the physical time is a parameter, which can quantize itself because energy, as we know, also quantizes.

Using F. (2), we can show why the time for the old peoples going more quickly than for the younger human beaning's.

Write down the expression (2) for a certain process in our Universe:

$$
\begin{gathered}
\Delta E^{\prime}=M^{\prime} \Delta T^{\prime} ; \Delta E>>>\Delta E^{\prime} ; \Delta T^{\prime}=\Delta T+\Delta T^{\prime \prime} ; \Delta T>>>\Delta T^{\prime \prime}, \\
M^{\prime}<<<M, \\
\Delta T=\frac{\Delta E}{M}, \Delta T^{\prime}=\Delta T+\Delta T^{\prime \prime}=\frac{\Delta E^{\prime}}{M^{\prime}} .
\end{gathered}
$$


Taking into consideration (6) - (8) we can see that the second equation from (6) can be observed.

\section{CONCLUSIONS}

1) The physical parameter time $T$ has material nature.

2) The principle of equivalence was formulated of energy spent on a process and time.

3) Physical time can quantize like energy.

\section{REFERENCES}

Nitza Arbel, Zev Levin. \& quot ; (1977) The coalescence of water drops I. A theoretical model of approaching drops \& quot;, Pure and Applied Geophysics PAGEOPH. Retrieved from https://doi.org/10.1007/BF00881214

Veitsman, E.V. (2019) Long-ranged Interactions, Forces and Real Spaces Related to them Including Anisotropic Cases. J. Mathematics and Statistics, 7(5), 207 214. Retrieved from https://doi.org/10.13189/ms.2019.070506

Veitsman, E.V. (2002) Some Problem of Interface Chemical Mechanics. J. Colloid Interface Sci. $253 \quad$ (1), $103 \quad$ - 111. Retrieved from https://doi.org/10.1006/jcis.2002.8431

Veitsman. E.V. (2021) Veitsman's Theorem on Spaces and Forces forming them, Time and Chaos. J., Researcher-GANTHAALAYAN,8(06). 26 - 29. Retrieved from https://doi.org/10.29121/granthaalayah.v8.i6.2020.349

Xaase, R. (1955) Thermodynamik der Irreversiblen Prozesse, DoctorSteinkopf Verlag. Darmstadt 